\title{
Role of physiotherapy in hemophilia patient: a case study on new dimension of physiotherapy application
}

\begin{abstract}
Haemophilia is a blood-clotting disorder caused by a deficiency in factor VIII or factor IX. Joint bleeding is common feature and make patient disable if untreated. Physiotherapy is evidence based medical treatment to keep patient active. This 14 years old boy has been referred to me by a hematologist for physiotherapy. History taking, physical examination of joint and muscles, analysis of pain by visual analogue scale were my primary step. Then physiotherapy was given according to condition of patient. I have tried to take a thorough medical history from him and his parents. As he was kept in touch for long days, it was easy for me to enter in depth. Finally he was cured, active and back to normal life by dint of physiotherapy. Physiotherapy should be applied cautiously in case of haemophilia patient. In order to achieve good result physiotherapist have to know about details of hemophilia. This case can be an excellent example indeed.
\end{abstract}

Volume 2 Issue 3 - 2017

\author{
Easmin Ara Doly \\ Chief Physiotherapist, Firoza Bari Disabled Children Hospital, \\ Bangladesh
}

Correspondence: Easmin Ara Doly, Chief Physiotherapist, Firoza Bari Disabled Children Hospital, Bangladesh, Email gpabdI7@gmail.com

Received: August 02, 2017 | Published: August 16,2017

Keywords: hemophilia, physiotherapy, management

Abbreviations: FFP, fresh frozen plasma; BSMMU, bangabandhu sheikh mujib medical university

DMC, Dhaka medical college; PWH, person of world hemophilia; VAS, visual analogue scale

\section{Introduction}

Hemophilia is not a common disease. It is a mostly inherited genetic disorder that impairs the body's ability to make blood clots, a process needed to stop bleeding. It is a hereditary bleeding disorder caused by a lack or absent of blood clotting factor - VIII or IX. There are two main types of haemophilia: haemophilia A, which occurs due to not enough clotting factor VIII, and haemophilia B, which occurs due to not enough clotting factor IX). Joint bleeding is common in hemophilia followed by muscle bleeding, other bleeding and brain hemorrhage. The prevalence of hemophilia is estimated to be about 1:10000 birth and that of the severe form of the disease to be about $6 \%$ per 100000 population. ${ }^{1}$ Even medical personnel are sometimes not familiar with its diagnosis and management which is pathetic. As such many health care professionals are not aware of the integral role physiotherapists play in the management of people with bleeding disorders or sometimes they refer patient to other medical professional for physiotherapy. Lack of referral system makes this problem more severe though scenario is changing day by day. Seminar, symposium, conference and workshop regarding Haemophilia can build up awareness. Physiotherapy is recognized as a mean to improve quality of life, improve healing times and decrease frequency of bleeds, and to aid in the prevention of joint destruction due to multiple bleeds.

\section{Case history}

Master Abdullah- 14years old boy is the subject under study. At his first years of birth, he used to have bruises often all over his body. They would disappear often a few days. When he was 3years old, he fell from a chair and his mouth struck at the brain of a vessel on the floor. He was taken to Bangabandhu Sheikh Mujib Medical University (BSMMU) where his condition was diagnosed as Hemophilia-A at a severe level, less than $1 \%$. He had frequent bleeding in the right knee joint and sometime gum bleeding. At the age of 5years he had a major bleed in the urinary track and developed Hematuria. His left knee had become stiff due to prolonged bleeds and also because of a fall few years ago. The knee joint had been dislocated. He used to treat with pain killer, ice, Fresh Frozen Plasma (FFP). He started receiving factor replacement since a few years. He was admitted to school at the age of 6yrs. He had to change school since the management refused to take any care about him. At present he has no problem with teachers or schoolmates on account of hemophilia, but now he faces some problem or non-co-operation from his family members specially his father. His father thinks or believes that "It is a non-curative disease and his wife is responsible for the disease" so he is not interested to do any treatment or other co-operation even not continue education. Sometimes he also avoids his wife. From January 2017, he has been suffering recurrent bleeding in his right knee joint. Usually he has treated by FFP and other medication advised by hematologist of Dhaka medical College (DMC) and BSMMU. Very recently one of hematologist referred him at Firoza Bari Disabled Children Hospital for physiotherapy. Firoza Bari Disabled Children Hospital is one of the re-known rehabilitation residential center for hemophilia. As a chief and concern physiotherapist my duty was to assess him and found quadriceps muscles weakness, restricted joint movement and pathological dislocation of patella is the main causes of recurrent bleeding. Faradic muscles stimulation was applied over the right quadriceps longitudinally up to visible contraction followed by ultra sound therapy in $0.8 \mathrm{~m} / \mathrm{cm}^{2}$ over same area and gentle mobilizing exercise. After 7days encouraged him to start free active knee flexion and extension in gravity eliminated position. Within 15 days he gained a confident and started to do exercise in against gravity to increase muscle power. He is advised to start progressive muscles strengthening exercises, but within a week he noticed me that it was not possible because of pain. In this situation I decided to stop all exercises and 
advised him to applied ice 15 mins every 2 hourly followed by rest After 3days he was feeling better. After re-assessment of his joint and muscles condition and decided to start isometric muscles exercises first, then progressive muscles strengthening exercises. After 2 months he gained grade-5 Quadriceps muscles power and able to do knee flexion and extension with maximum resistance. Now he is 14years old. He has been working in a departmental shop as a part time sales man along with his education. He spends his earning money to continue his education. Last 11years I have been treated about 600 PWH (Person of World Hemophilia). Most of them leading a sound life by receiving regular Physiotherapy. Some of them minimized their disability by regular physiotherapy. So it is an extensive area of research and depth and large scale study can explore real picture.

\section{Discussion}

Hemophilia is managed by holistic approach. Educating patient and his family (parents) about nature of the disease, replacement of clotting factor and comprehensive care. Evidence based therapeutic exercise and proper nutrition can help in controlling bleeding and maintain general health. Focus should be primary prevention of bleeding as well as treatment of acute bleeding otherwise life will be risky.

Primary aims are- prevention of bleeding and treatment of acute bleeding. Multidisciplinary team approach can be instituted to bring efficient result. Home therapy, attention for psychosocial health and rehabilitation program is an aid to restore joint and ensure mobility. ${ }^{2}$ This case shows that misconception about disease is present among his father and lack of family support. Evidence supports that management of hemophilia in developing countries poses great challenges. Government and family monetary resources are usually inadequate, knowledge and awareness about hemophilia and its management often nonexistent. ${ }^{3}$ Literature suggests that hospital based studies did not report any case detected in newborn period reflecting inadequacy of knowledge and attitude of the treating physician towards haemophilia. ${ }^{3}$ A study was carried out to evaluate the progress of musculoskeletal ability and pain in haemophilia $\mathrm{A}$ and $\mathrm{B}$ patients during a given period of time and to find out the role of rehabilitation and physiotherapy in this process. In the physiotherapy/ rehabilitation group, pain index by visual analogue scale (VAS) improved through time by $26 \% / 4.6 \%$. The non-treatment group showed slight aggravation. Regression analysis revealed that age, pain intensity, and functional disability in 1994 were the only significant explanatory factors influencing musculoskeletal functional ability at the end of the study. Positive effect of physiotherapy and inpatient rehabilitation courses in reducing pain and functional disability for haemophilia patients was proven. One of the important contributions of physiotherapy and rehabilitation is their educational role. ${ }^{4}$ Actually therapeutic exercises and electrotherapy equipment was applied in the present case wisely and it is proven effective. After treatment he gained grade-5 quadriceps muscles power and able to do knee flexion and extension with maximum resistance and without feel any pain and discomfort. Though it is a case study and it is very much difficult to take concrete decision from a single case but it gives some hints. If available more similar cases can be taken to draw case series.

\section{Conclusion}

Physiotherapy has immense role in treating hemophilia patient. From this particular case it can be concluded that in case of pain reduction as well as restore joint mobility physiotherapy plays vital role. It is not wise to apply same treatment plan for all. Treatment should not be generalized. Physiotherapy plan should be individualized.

\section{Acknowledgements}

None.

\section{Conflict of interest}

As this is a single author, there is no conflict of interest in this work.

\section{Source of funding}

The author did not receive any source of fund or grant to conduct the case study.

\section{References}

1. Islam S, Morshed AKMA, Khan ZJ. Hemophilia in Children. J Paediatr Surg Bangladesh. 2015;6(2):54-63.

2. Srivastava A, Brewer AK, Mauser Bunschoten EP, et al. WFH guidelines for the management of hemophilia. Haemophilia. 2013;19(1):1-47.

3. Pipe SW. The hope and reality of long acting hemophilia products. $A m J$ Hematol. 2012;87(suppl 1):S33-39.

4. Santavirta N, Solovieva S, Helkama O, et al. Musculoskeletal pain and functional ability in haemophilia A and B. Physiotherapy and rehabilitation in haemophilia patients. Rheumatol Int. 2001;21(1):15-19. 